\title{
Cryptotanshinone induces reactive oxygen species-mediated apoptosis in human rheumatoid arthritis fibroblast-like synoviocytes
}

\author{
HU-NAN SUN ${ }^{1 *}$, YING-HUA LUO $^{2 *}$, LING-QI MENG $^{1 *}$, XIAN-JI PIAO ${ }^{3}$, \\ YUE WANG $^{1}$, JIA-RU WANG ${ }^{1}$, HAO WANG $^{1}$, YI ZHANG ${ }^{1}$, JIN-QIAN LI ${ }^{1}$, WAN-TING XU ${ }^{1}$, \\ YANG LIU $^{1}$, YU ZHANG ${ }^{1}$, TONG ZHANG ${ }^{1}$, YING-HAO HAN ${ }^{1}$, MEI-HUA JIN ${ }^{1}$, GUI-NAN SHEN ${ }^{1}$, \\ YAN-QING ZANG ${ }^{4}$, LONG-KUI CAO ${ }^{4}$, DONG-JIE ZHANG ${ }^{4}$ and CHENG-HAO JIN ${ }^{1,4}$ \\ ${ }^{1}$ Department of Biochemistry and Molecular Biology, College of Life Science and Technology, \\ Heilongjiang Bayi Agricultural University; ${ }^{2}$ Department of Grass Science, College of Animal Science and \\ Veterinary Medicine, Heilongjiang Bayi Agricultural University, Daqing, Heilongjiang 163319; ${ }^{3}$ Department of \\ Gynaecology and Obstetrics, The Fifth Affiliated Hospital of Harbin Medical University, Daqing, Heilongjiang 163316; \\ ${ }^{4}$ Department of Food Science and Engineering, College of Food Science and Technology, \\ Heilongjiang Bayi Agricultural University, Daqing, Heilongjiang 163319, P.R. China
}

Received May 3, 2018; Accepted November 16, 2018

DOI: $10.3892 /$ ijmm.2018.4012

\begin{abstract}
The present study investigated the mechanisms of apoptosis induced by cryptotanshinone (CT) in human rheumatoid arthritis fibroblast-like synoviocytes (RA-FLSs). Cell Counting kit-8 assay was performed to determine the cytotoxic effects of CT in human RA-FLSs, including primary RA-FLS, HFLS-RA and MH7A cells, and in HFLS cells derived from normal synovial tissue. Annexin V-FITC/PI staining was used to detect the apoptotic effects of CT in HFLS-RA and MH7A cells. Flow cytometry was performed to detect the apoptotic and reactive oxygen species (ROS) levels induced by CT in HFLS-RA cells. Western blotting was used to assess the expression levels of proteins associated with apoptosis and with the mitogen-activated protein kinase (MAPK), protein kinase B (Akt), and signal transducer and activator of transcription-3
\end{abstract}

Correspondence to: Professor Cheng-Hao Jin, Department of Biochemistry and Molecular Biology, College of Life Science and Technology, Heilongjiang Bayi Agricultural University, 5 Xinfa Street, Daqing, Heilongjiang 163319, P.R. China

E-mail: jinchenghao3727@qq.com

Professor Dong-Jie Zhang, Department of Food Science and Engineering, College of Food Science and Technology, Heilongjiang Bayi Agricultural University, 5 Xinfa Street, Daqing, Heilongjiang 163319, P.R. China

E-mail: byndzdj@126.com

${ }^{*}$ Contributed equally

Key words: cryptotanshinone, rheumatoid arthritis fibroblast-like synoviocytes, apoptosis, reactive oxygen species
(STAT3) signaling pathways. The results demonstrated that CT treatment significantly suppressed HFLS-RA and MH7A cell growth, whereas no clear inhibitory effect was observed in normal HFLS cells. CT exposure downregulated the expression levels of B-cell lymphoma 2 (Bcl-2), p-Akt, p-extracellular signal-related kinase and p-STAT3, while it upregulated the expression levels of Bcl-2-associated death promoter (Bad), caspase-3, poly (ADP-ribose) polymerase (PARP), p-p38 and p-c-Jun N-terminal kinase. Following ROS scavenging, the CT-induced apoptosis and altered expression levels of Bcl-2, Bad, cleaved caspase- 3 and cleaved PARP were restored. Furthermore, the Akt, MAPK and STAT3 signaling pathways were regulated by intracellular ROS. These results suggest that ROS-mediated Akt, MAPK and STAT3 signaling pathways serve important roles in the CT-induced apoptosis of RA-FLSs.

\section{Introduction}

Rheumatoid arthritis (RA) is a common systemic and autoimmune inflammatory disease of the joints, characterized by inflammation, synovial hyperplasia and abnormal immune responses (1-3). Fibroblast-like synoviocytes (FLSs) in the RA synovium (referred to as RA-FLSs) may affect the vascular endothelium (4). Furthermore, accumulating evidence has suggested that RA-FLSs serve crucial roles in the destruction process of the joints and the development of RA (5-7). RA-FLSs have aggressive properties similar to those exhibited by cancer cells, including hyperproliferation, defective apoptosis and invasiveness (8). Thus, the prevention of RA-FLS proliferation and induction of apoptosis may be an effective therapeutic strategy for the treatment of RA.

Protein kinase B (Akt) is a serine/threonine kinase and downstream effector of phosphoinositide 3-kinase 
(PI3K). Activated PI3K increases the intracellular content of phosphatidylinoside-4,5-diphosphate and phosphatidylinoside-3,4,5-triphosphate, positively inducing Akt phosphorylation (9). The mitogen-activated protein kinase (MAPK), extracellular signal-regulated kinase (ERK), c-Jun N-terminal kinase (JNK) and p38 signaling pathways are constructed around a three-tiered phosphorylation cascade. The MAPK signaling pathway-associated proteins are known to be involved in the mediation of inflammation, and serve a critical role in the excessive proliferation and invasion of synovial fibroblasts of RA (10-12). Janus kinase (JAK) activation leads to the activation of downstream substrates and of signal transducer and activator of transcription (STAT) proteins, followed by their nuclear translocation and subsequent activation of target genes (13). The STAT3 signaling pathway is responsible for transmitting the outer signals of the cell to the nucleus and expressing the effects of biological stimulation through the induction of target gene transcription. Dysfunctional JAK/STAT signaling has been implicated in various hematological and immunological disorders, as well as other pathological inflammatory conditions (14-16). Reactive oxygen species (ROS), including oxygen ions, peroxides and oxygen-free radicals, are the main molecules that the body produces during oxidative stress. Highly reactive oxygen-free radicals are considered to be involved in the pathogenesis of RA (17-21).

The use of traditional Chinese medicine is promising for adjuvant therapy due to the low cost and low toxicity of the formulations used (22). Cryptotanshinone (CT) is a fat-soluble anthraquinone derivative and a major tanshinone found in Salvia miltiorrhiza Bunge (Lamiaceae) (17). It has a wide range of pharmacological properties, such as antitumor, anti-bacterial and anti-inflammatory properties (22-25). A number of studies have reported that $\mathrm{CT}$ inhibits the growth of certain types of cancer cells or induces cancer cell apoptosis, including in lung, breast and prostate cancer (26-29). However, the effects of CT on the proliferation and apoptosis of RA-FLSs, and the underlying molecular mechanisms remain unclear.

In the present study, the effects of CT on RA-FLS proliferation and apoptosis were evaluated, and the associated molecular pathways were examined. In addition, the roles of the MAPK, Akt and STAT3 signaling pathways associated with ROS generation were investigated in RA-FLSs.

\section{Materials and methods}

Chemicals and reagents. CT (Sigma-Aldrich; Merck KGaA, Darmstadt, Germany) was dissolved (20 mM CT in $100 \%$ dimethyl sulfoxide) to obtain a stock solution and stored at $-20^{\circ} \mathrm{C}$. The stock solution was diluted with cell culture media prior to further use.

Patients and samples. Informed consent was obtained from RA patients prior to inclusion in the present study, and the procedure was approved by the Ethical Committee of the Fifth Affiliated Hospital of Harbin Medical University (Daqing, China). Synovial tissue samples were obtained from RA patients (including 3 females and 3 males aged 53-66 years) undergoing synovectomy or joint replacement in the Fifth Affiliated Hospital of Harbin Medical University. The synovial tissues were minced into small pieces and digested with
$2 \mathrm{mg} / \mathrm{ml}$ collagenase type I at $37^{\circ} \mathrm{C}$ for $4 \mathrm{~h}$. Subsequent to the enzymatic dispersion of synovial tissues, synovial cells were pelleted by centrifugation $(1,200 \mathrm{x} \mathrm{g}$ for $3 \mathrm{~min}$ at room temperature) and cultured in Dulbecco's modified Eagle's medium (DMEM; Gibco; Thermo Fisher Scientific, Inc., Waltham, MA, USA) at $37^{\circ} \mathrm{C}$ in a humidified atmosphere containing $5 \% \mathrm{CO}_{2}$. After $24 \mathrm{~h}$ of incubation, the adherent cells were collected and cultured in DMEM, and are referred to as the primary RA-FLS cells.

Cell lines and culture. The HFLS-RA (cat. no. CBR130803), MH7A (cat. no. CBR130742) and normal synovial HFLS (cat. no. CBR130948) cells were purchased from Saiqi Biological Engineering Co., Ltd. (Shanghai, China). Cells were cultured in DMEM supplemented with $10 \%$ fetal bovine serum (Gibco; Thermo Fisher Scientific, Inc.), $100 \mu \mathrm{g} / \mathrm{ml}$ penicillin and $100 \mathrm{U} / \mathrm{ml}$ streptomycin in a humidified $5 \% \mathrm{CO}_{2}$ incubator at $37^{\circ} \mathrm{C}$.

Cell Counting kit-8 (CCK-8) proliferation test. Primary RA-FLS, HFLS-RA, MH7A and HFLS cells were seeded on 96-well plates $\left(1 \times 10^{4}\right.$ cells/well) and treated with different concentrations of CT $(1,3,10,30$ and $100 \mu \mathrm{mol} / \mathrm{l})$ for $24 \mathrm{~h}$. Prior to proliferation detection, $10 \mu \mathrm{lCCK}-8$ reagent (Beyotime Institute of Biotechnology, Shanghai, China) was added into each well and incubated at $37^{\circ} \mathrm{C}$ for $1.5 \mathrm{~h}$. Finally, the absorbance was measured by a microplate reader $\left(\mathrm{A}_{490}\right)$ and the cell survival rates were calculated.

Fluorescence microscopy. A total of $5 \times 10^{5}$ HFLS-RA and MH7A cells/well were seeded in 6-well plates and cultured overnight. Next, the cells were treated with $5 \mu \mathrm{mol} / \mathrm{l} \mathrm{CT}$ for different time durations $(0,3,6,12$ and $24 \mathrm{~h})$. According to the manufacturer's manual that was provided with the Apoptosis Detection kit (Beyotime Institute of Biotechnology), cells were stained with $10 \mu \mathrm{l}$ Annexin V-FITC and $5 \mu \mathrm{l}$ propidium iodide (PI) for $15 \mathrm{~min}$, and the fluorescence intensity of each sample was determined by fluorescence microscopy (Leica Microsystem, Wetzlar, Germany).

Flow cytometry. A total of $5 \times 10^{5}$ HFLS-RA were seeded in 6-well plates and cultured overnight. HFLS-RA cells were pretreated with $\mathrm{N}$-acetyl cysteine (NAC, $5 \mathrm{mmol} / \mathrm{l}$, Sigma-Aldrich; Merck KGaA) for $30 \mathrm{~min}$ and incubated with CT $(5 \mu \mathrm{mol} / \mathrm{l})$ for $24 \mathrm{~h}$. Then, cells were stained with $10 \mu \mathrm{l}$ Annexin V-FITC and $5 \mu \mathrm{l}$ PI for $15 \mathrm{~min}$, and the number of apoptotic cells were detected by flow cytometry (Beckman Coulter, Inc., Brea, CA, USA).

In order to measure the ROS levels, HFLS-RA cells were treated with CT (5 $\mu \mathrm{mol} / \mathrm{l})$ for $0,3,6,12$ and $24 \mathrm{~h}$, following which the cells were incubated with 2',7'-dichlorofluorescein-diacetate (DCFH-DA; Merck, Shanghai, China) for $30 \mathrm{~min}$ at $37^{\circ} \mathrm{C}$. Next, cells were washed 2-3 times with PBS and harvested by centrifugation at $1,200 \mathrm{x} \mathrm{g}$ for $5 \mathrm{~min}$ at room temperature. The intracellular ROS levels were subsequently analyzed by flow cytometry.

Western blot analysis. Cells were lysed in ice-cold radioimmunoprecipitation buffer (50 mmol/1 Tris (pH 7.4), $150 \mathrm{mmol} / \mathrm{l}$ $\mathrm{NaCl}, 1 \%$ Triton $\mathrm{X}-100,1 \%$ sodium deoxycholate, $0.1 \%$ SDS, 
A

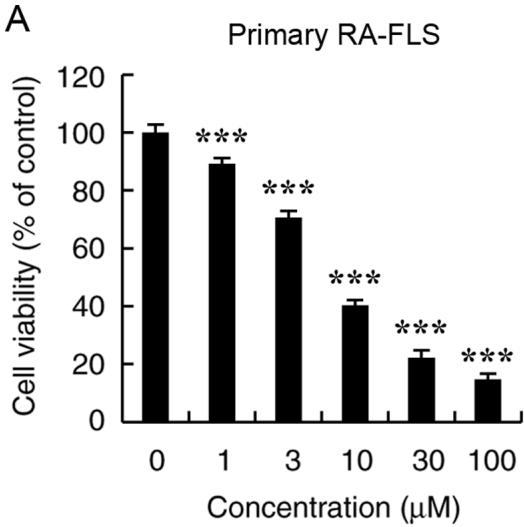

C

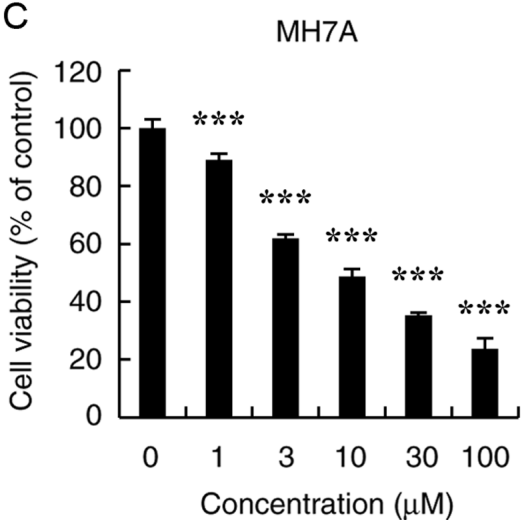

B

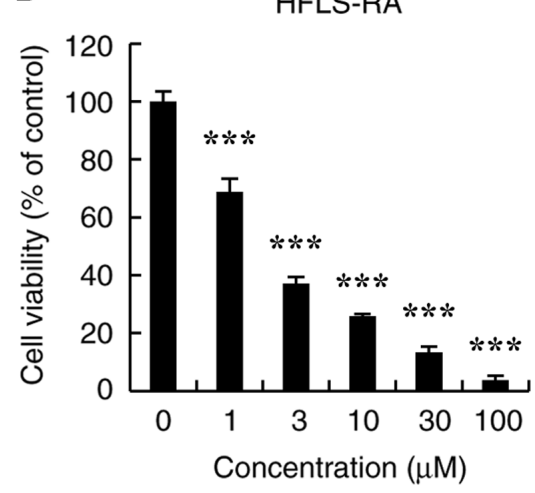

D

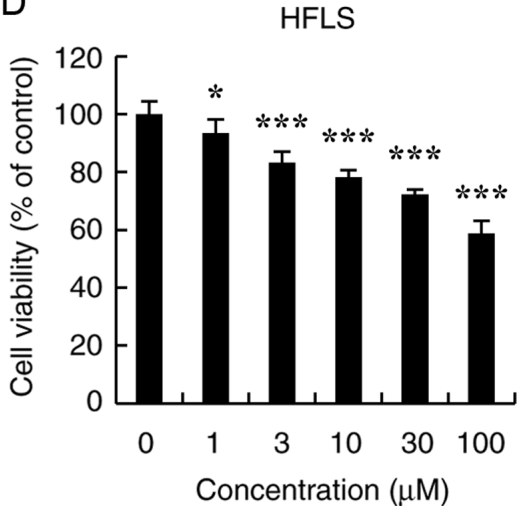

Figure 1. Effects of CT on the proliferation of primary RA-FLS, HFLS-RA, MH7A and HFLS cells. Cells were treated with different concentrations of CT $(0,1,3,10,30$ and $100 \mu \mathrm{mol} / \mathrm{l})$ for $24 \mathrm{~h}$, following which the cell viability was evaluated by a Cell Counting kit- 8 assay. The cytotoxic effects of CT on (A) Primary RA-FLS, (B) HFLS-RA, (C) MH7A and (D) HFLS cell viability are displayed. ${ }^{*} \mathrm{P}<0.05$ and ${ }^{* * * *} \mathrm{P}<0.001$, vs. control (0 $\mu$ mol/1 CT) group. CT, cryptotanshinone.

$20 \mathrm{mg} / \mathrm{ml}$ AEBSF, $0.5 \mathrm{mg} / \mathrm{ml}$ pepstatin, $0.5 \mathrm{mg} / \mathrm{ml}$ leupeptin and $2 \mathrm{mg} / \mathrm{ml}$ aprotinin; (Beyotime Institute of Biotechnology) at $4^{\circ} \mathrm{C}$ for $30 \mathrm{~min}$ by shaking every $5 \mathrm{~min}$ and then separated by centrifugation at $4^{\circ} \mathrm{C}$ and $12,000 \mathrm{x}$ for $30 \mathrm{~min}$. The protein concentrations were determined using Bradford reagent (Bio-Rad Laboratories, Inc., Hercules, CA, USA). Next, 5X buffer was added to the total supernatants $(30 \mu \mathrm{g})$, and proteins were resolved by $12 \%$ sodium dodecyl sulfate-polyacrylamide gel electrophoresis. Subsequently, the proteins were transferred to nitrocellulose (NC) membranes, which were blocked in $5 \%$ skim milk for $2 \mathrm{~h}$ at room temperature. The $\mathrm{NC}$ membranes were then incubated overnight at $4^{\circ} \mathrm{C}$ with the following primary antibodies (all purchased from Santa Cruz Biotechnology, Inc., Dallas, TX, USA): Mouse monoclonal antibodies against $\alpha$-tubulin (1:2,500; cat. no. sc-8035), B-cell lymphoma 2 (Bcl-2; 1:1,500; cat. no. sc-7382), Bcl-2-associated death promoter (Bad; 1:1,500; cat. no. sc-8044), cleaved caspase-3 (1:1,500; cat. no. sc-373730), cleaved poly (ADP-ribose) polymerase (PARP; 1:1,500; cat. no. sc-8007), phosphorylated ERK [p-ERK (Tyr 204); 1:1,500; cat. no. sc-8059], p-JNK (Thr 183 and Tyr 185, 1:1,500; cat. no. sc-6254), JNK (1:1,500; cat. no. sc-7345), p-p38 (1:1,500; cat. no. sc-7973), p-STAT3 (Tyr 705, 1:1,500; cat. no. sc-8059) and STAT3 (1:1,500; cat. no. sc-8019); and rabbit polyclonal antibodies against $\operatorname{ERK} 2$ (1:1,500; cat. no. sc-154), p38 $\alpha / \beta$ (1:1,500; cat. no. sc-7972), Akt1/2/3 (1:1,500; cat. no. sc-8312) and p-Akt1/2/3 (Ser 473, 1:2,500; cat. no. sc-7985-R). Following washing with Tris-buffered
saline/Tween 20 (TBST), the NC membranes were incubated with horseradish peroxidase (HRP)-labeled goat anti-rabbit $\operatorname{IgG}(1: 5,000$; cat. no. ZB-2301) and goat anti-rat IgG (1:5,000; cat. no. ZB-2305) were used as secondary antibodies for $2 \mathrm{~h}$ at room temperature. After the membranes were washed again with TBST, an enhanced chemiluminescence reagent (Thermo Fisher Scientific, Inc.) was used to detect the relative intensities of proteins using Amersham imager 600 (AI600; GE Healthcare, Chicago, IL, USA). The band intensities were analyzed by ImageJ software (National Institutes of Health, Bethesda, MD, USA), and the relative intensity of protein expression levels was calculated by SPSS version 18.0 statistical software (SPSS, Inc., Chicago, IL, USA).

Statistical analysis. Data are presented as the mean \pm standard deviation, and all the experiments were replicated three times. Continuous data were analyzed by one-way analysis of variance followed by Tukey's post hoc tests using SPSS version 18.0 statistical software. $\mathrm{P}<0.05$ was considered to indicate a difference that was statistically significant.

\section{Results}

$C T$ exhibits cytotoxic effects in primary RA-FLS, HFLS-RA and MH7A cells. To determine the effects of CT on RA-FLS survival, primary RA-FLS, HFLS-RA, MH7A and normal HFLS cells were treated with different concentrations of CT 
A
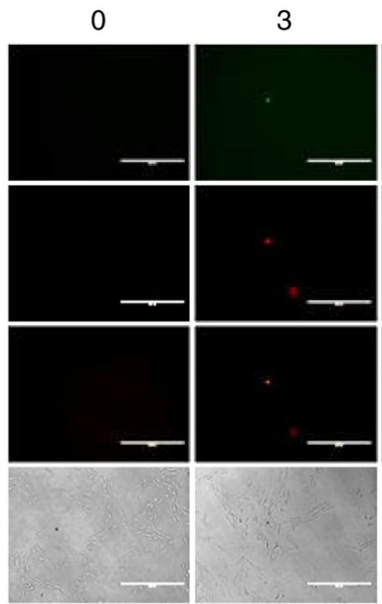

C
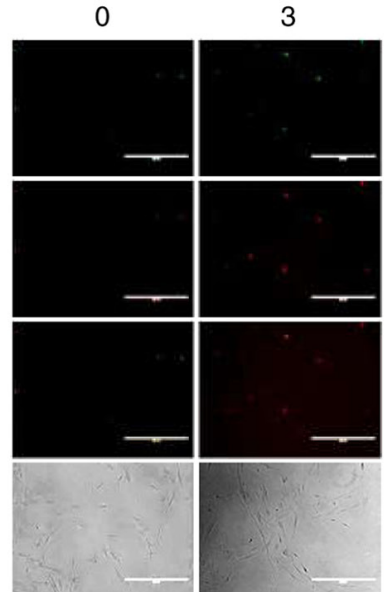

E
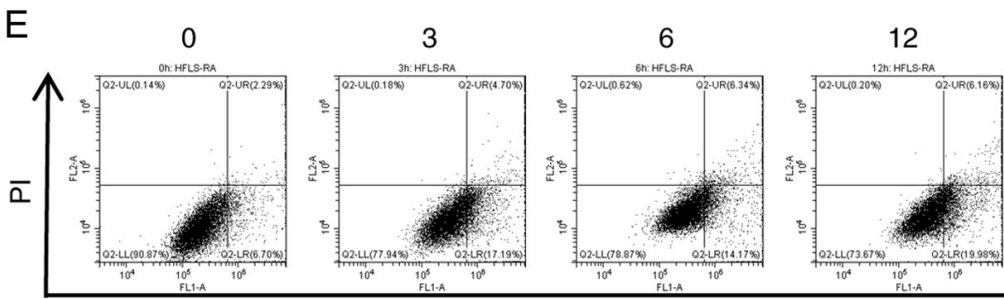

Annexin V-FITC

G
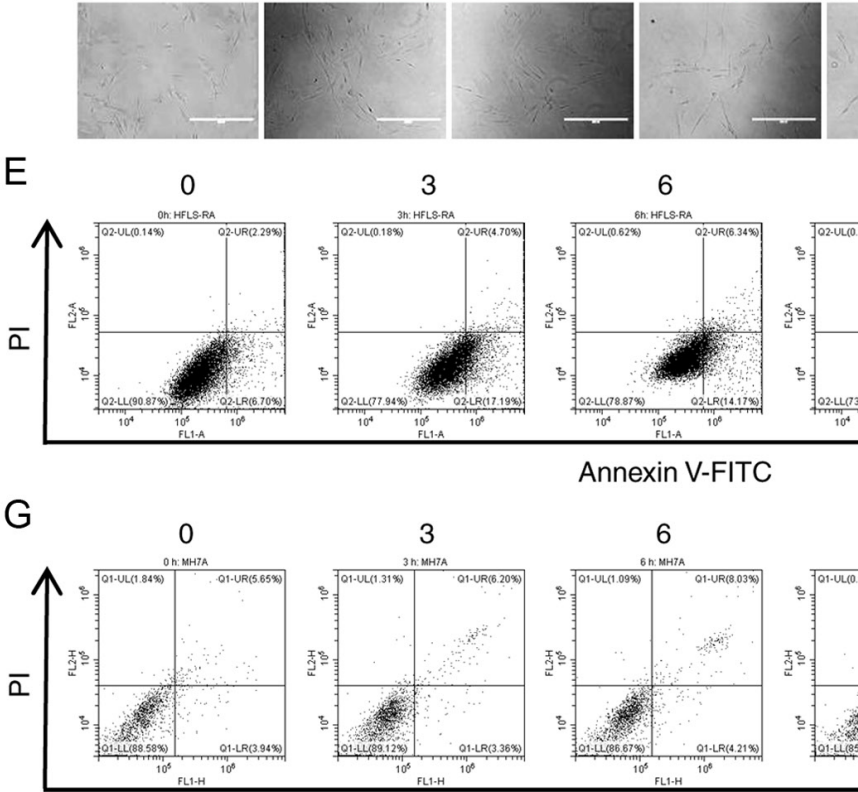
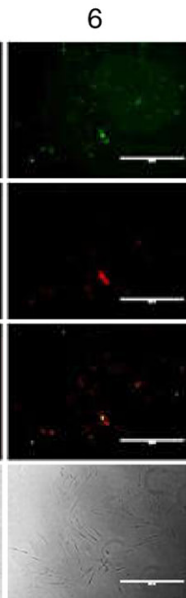

6
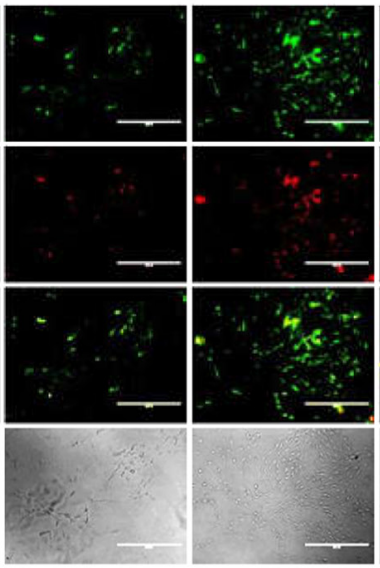

12
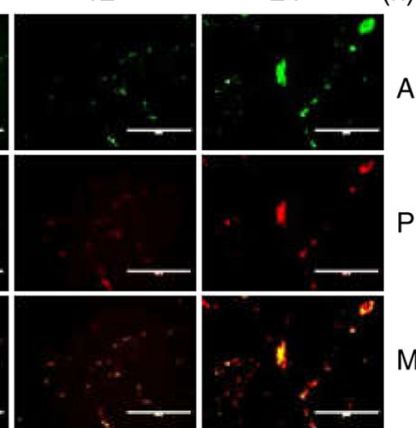

Merge
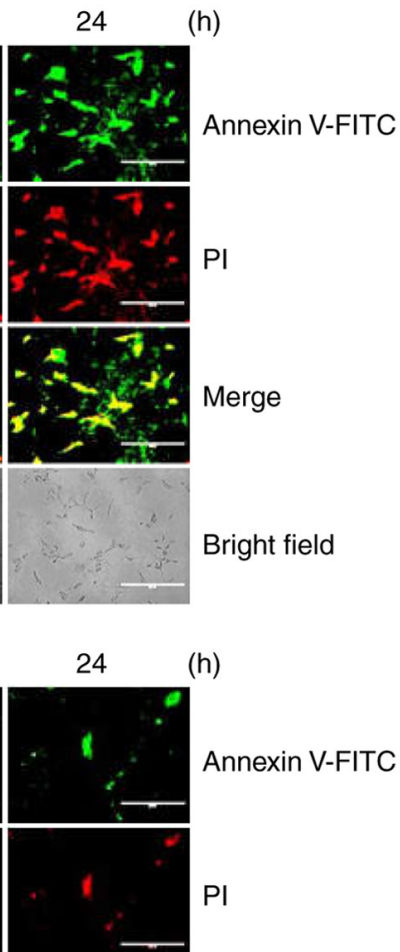

Bright field

Bright field

B
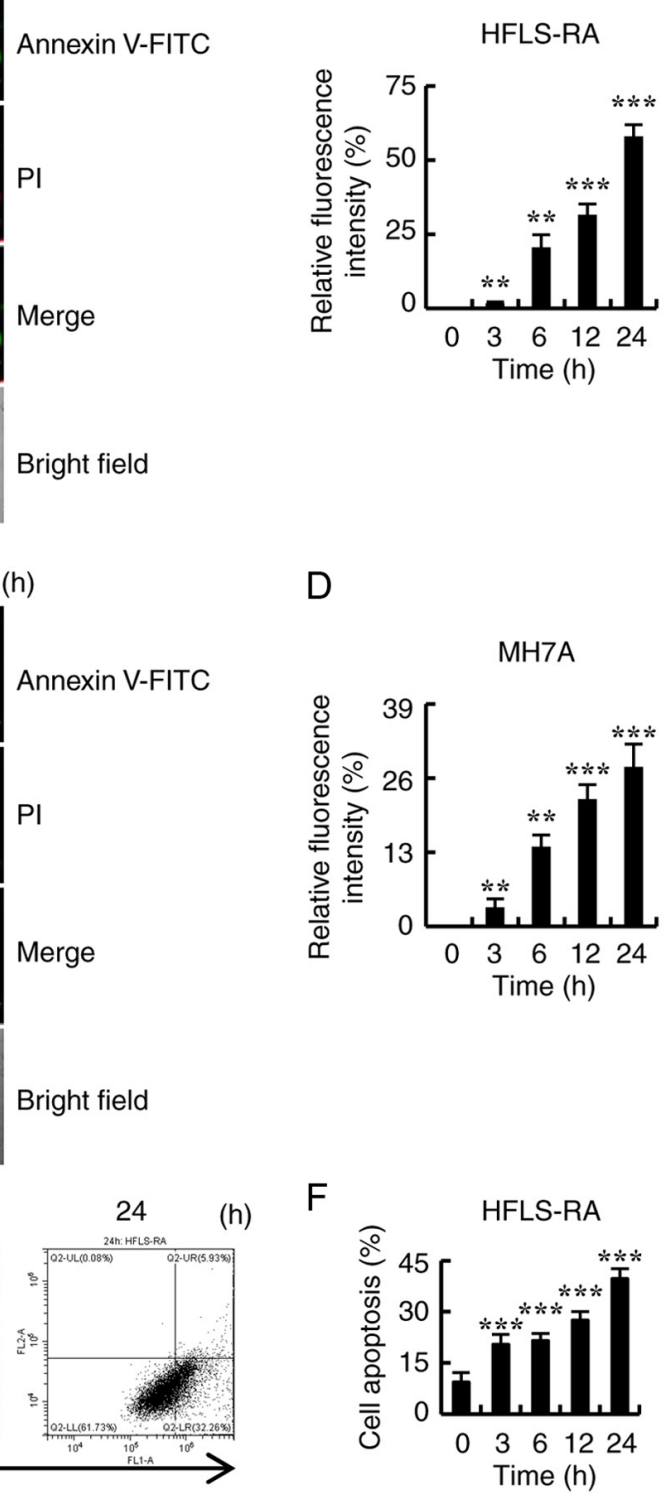

F

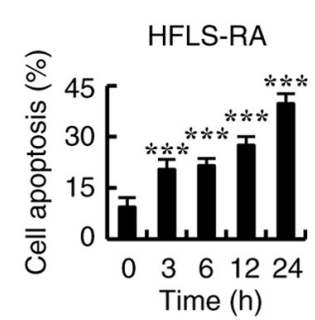

$\mathrm{H}$

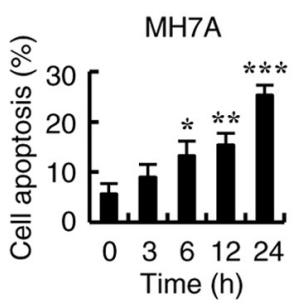

Figure 2. Effects of $\mathrm{CT}$ on the apoptosis of HFLS-RA and MH7A cells. Cells were treated with $5 \mu \mathrm{mol} / \mathrm{CT}$ for different time durations $(0,3,6,12$ and $24 \mathrm{~h})$. (A) Fluorescence microscopic images (magnification, x400; scale bars, $200 \mu \mathrm{m}$ ) and (B) quantified fluorescence intensity of HFLS-RA cells stained with Annexin V-FITC/PI. (C) Fluorescence microscopic images (magnification, x400; scale bars, $200 \mu \mathrm{m}$ ) and (D) quantified fluorescence intensity of MH7A cells stained with Annexin V-FITC/PI. (E) Flow cytometry results and (F) quantified cell apoptosis rate in HFLS-RA cells subsequent to staining with Annexin V-FITC/PI. (G) Flow cytometry results and (H) quantified cell apoptosis rate in MH7A cells subsequent to staining with Annexin V-FITC/PI. ${ }^{*} \mathrm{P}<0.05,{ }^{* *} \mathrm{P}<0.01$ and ${ }^{* * *} \mathrm{P}<0.001$, vs. control $(0 \mathrm{~h})$ group. $\mathrm{CT}$, cryptotanshinone.

$(1,3,10,30$ and $100 \mu \mathrm{mol} / \mathrm{l})$ for $24 \mathrm{~h}$, and the cell viabilities were evaluated by the CCK-8 assay. As shown in Fig. 1A-C, CT significantly decreased the viabilities of primary RA-FLS, HFLS-RA and MH7A cells in a dose-dependent manner. The half maximal inhibitory concentration $\left(\mathrm{IC}_{50}\right)$ values of primary RA-FLS, HFLS-RA and MH7A cells were 6.69 \pm 0.97 , $2.56 \pm 0.42$ and $7.10 \pm 0.94 \mu \mathrm{mol} / 1$, respectively. Furthermore, CT exhibited low cytotoxic effects on HFLS cells, which had an $\mathrm{IC}_{50}$ value $>100 \mu \mathrm{mol} / \mathrm{l}$; however, significance was observed compared with the control (Fig. 1D). 
A

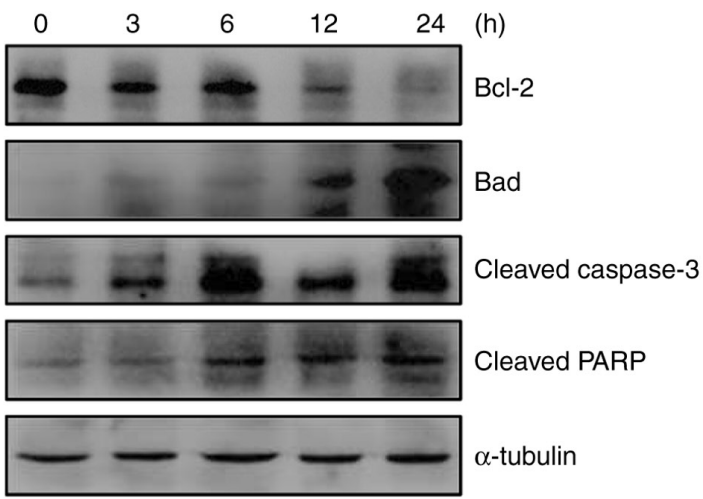

B
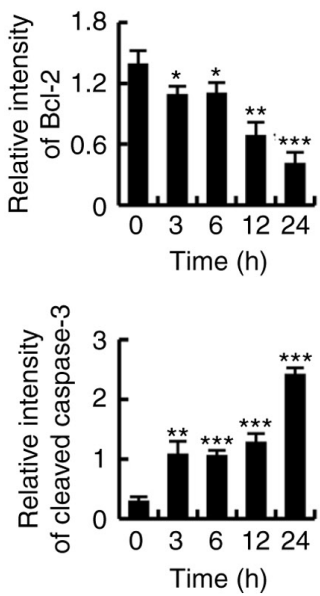
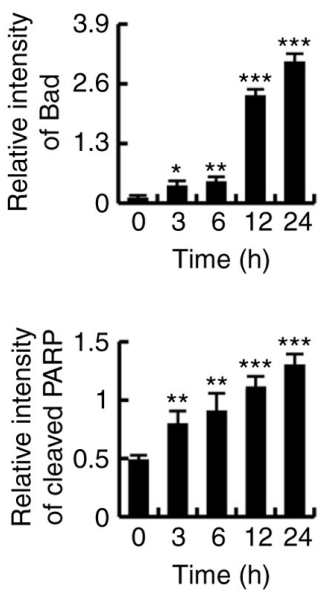

Figure 3. CT induced apoptosis by regulating the mitochondrial pathway in HFLS-RA cells. Cells were treated with $5 \mu$ mol/1 CT for various time durations $(0,3,6,12$ and $24 \mathrm{~h})$. (A) Western blot analysis results and (B) quantified protein expression levels of Bcl-2, Bad, cleaved caspase-3 and cleaved PARP in HFLS-RA cells. ${ }^{*} \mathrm{P}<0.05,{ }^{* *} \mathrm{P}<0.01$ and ${ }^{* * *} \mathrm{P}<0.001$, vs. control (0 h) group. CT, cryptotanshinone; Bcl-2, B-cell lymphoma 2; Bad, Bcl-2-associated death promoter; PARP, poly (ADP-ribose) polymerase.

A

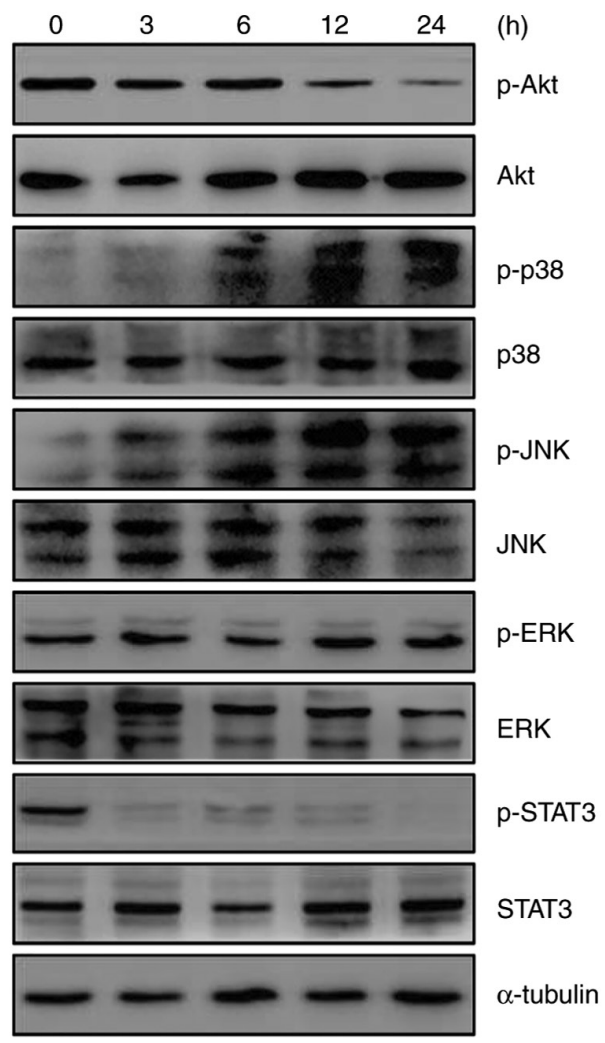

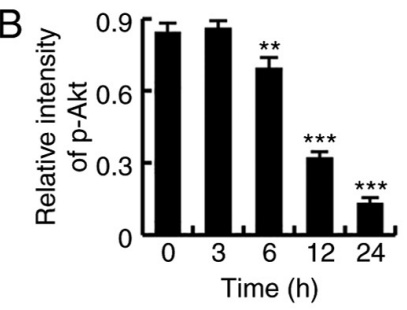
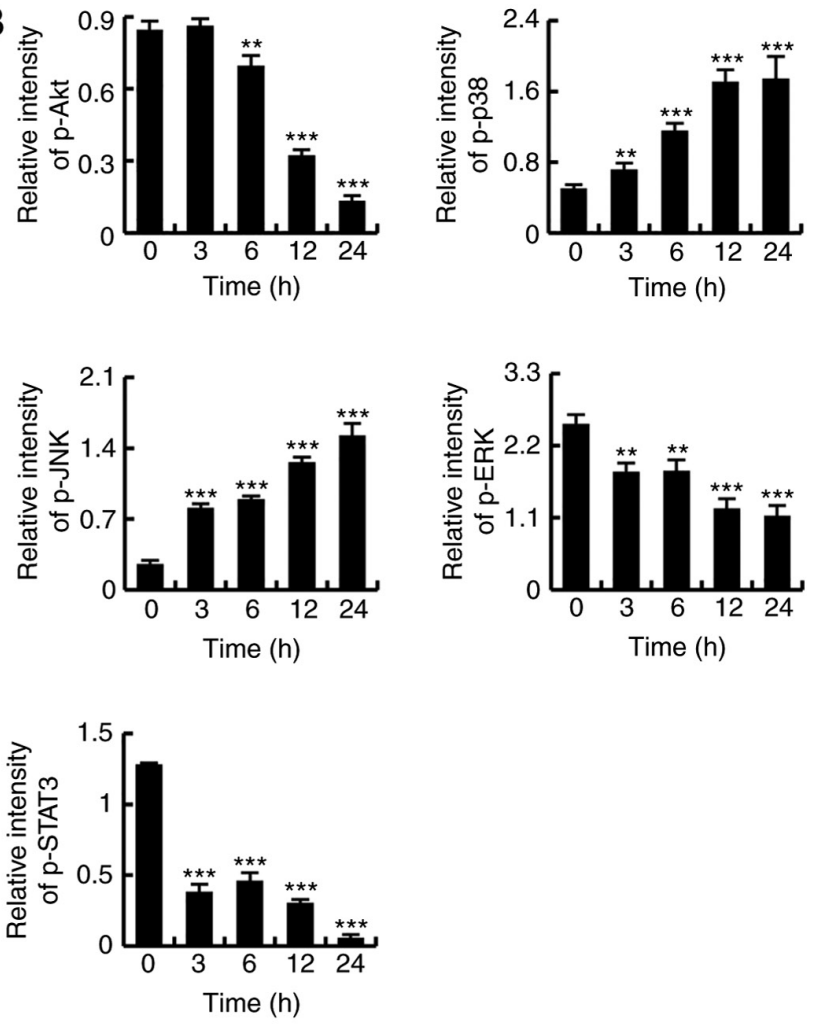

Figure 4. CT induced apoptosis by regulating the Akt, mitogen-activated protein kinase and STAT3 signaling pathways in HFLS-RA cells. Cells were treated with $5 \mu \mathrm{mol} / 1 \mathrm{CT}$ for various time durations $(0,3,6,12$ and $24 \mathrm{~h}$ ). (A) Western blot analysis results and (B) quantified protein expression levels of Akt, p38, JNK, ERK and STAT3. ${ }^{* *} \mathrm{P}<0.01$ and ${ }^{* * *} \mathrm{P}<0.001$, vs. control $(0$ h) group. CT, cryptotanshinone; Akt, protein kinase B; JNK, c-Jun N-terminal kinase; ERK, extracellular signal-regulated kinase; STAT3, signal transducer and activator of transcription-3; p-, phosphorylated.

CT induces apoptosis by regulating the mitochondrial pathways in HFLS-RA cells. To determine whether CT induced apoptosis in RA-FLSs, HFLS-RA and MH7A cells were treated with $5 \mu \mathrm{mol} / \mathrm{l}$ CT for different time points $(0,3,6,12$ and $24 \mathrm{~h})$, and apoptosis was examined by Annexin V-FITC/PI double staining and flow cytometry. As shown in Fig. 2A-D, the fluorescence intensity significantly increased with the increase in the duration of $\mathrm{CT}$ treatment.
In addition, apoptosis gradually increased in HFLS-RA and MH7A cells in a time-dependent manner (Fig. 2E-H). These results suggest that $\mathrm{CT}$ may be a potential inducer of apoptosis in RA-FLSs.

CT-induced apoptosis was more notable in HFLS-RA cells compared with in MH7A cells; thus, HFLS-RA cells were used as the representative RA-FLS cells in subsequent experiments. As shown in Fig. 3A and B, CT treatment markedly 

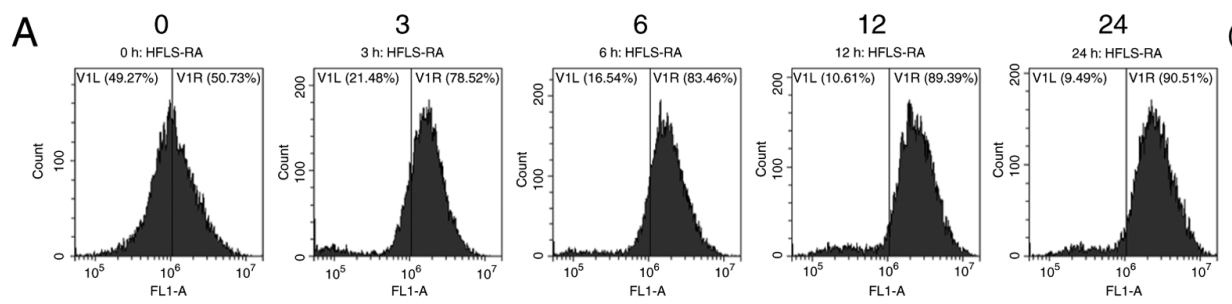

(h)
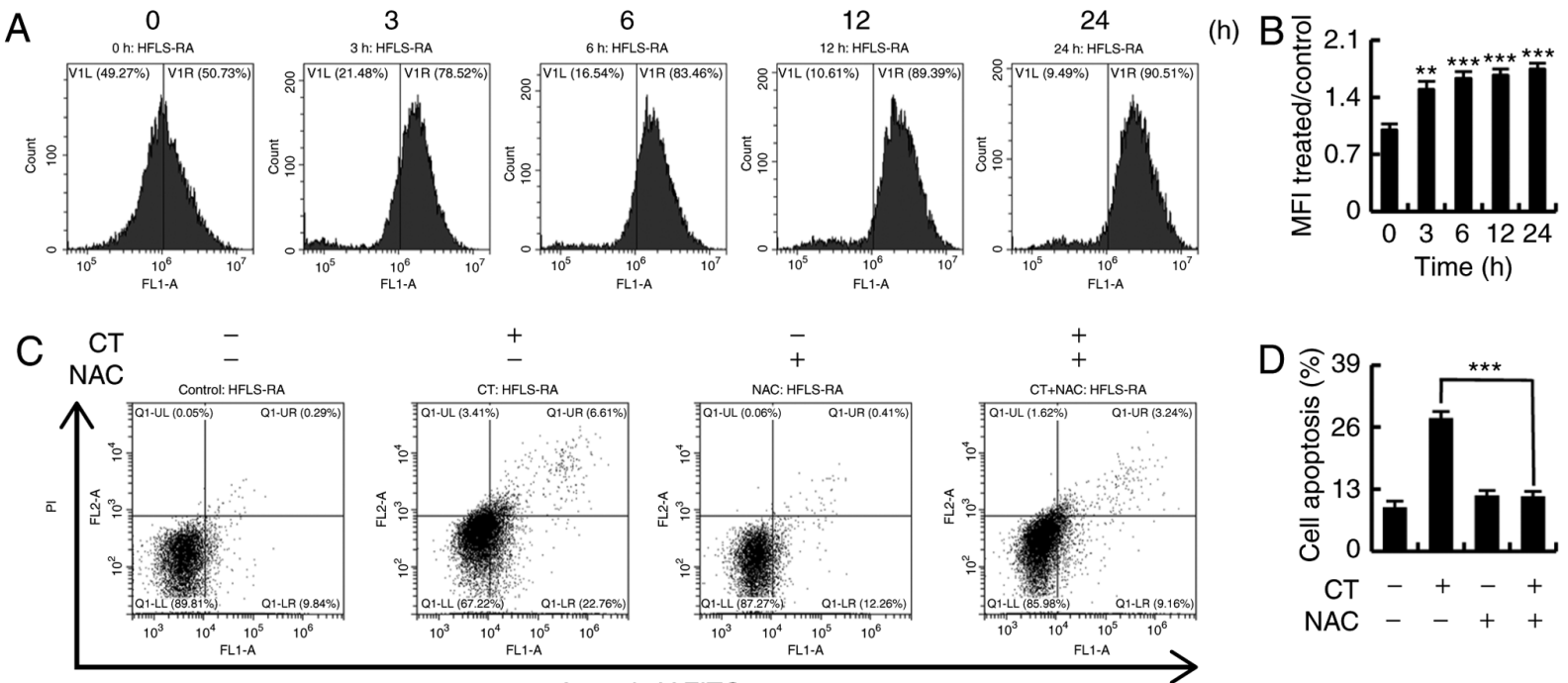

Annexin V-FITC
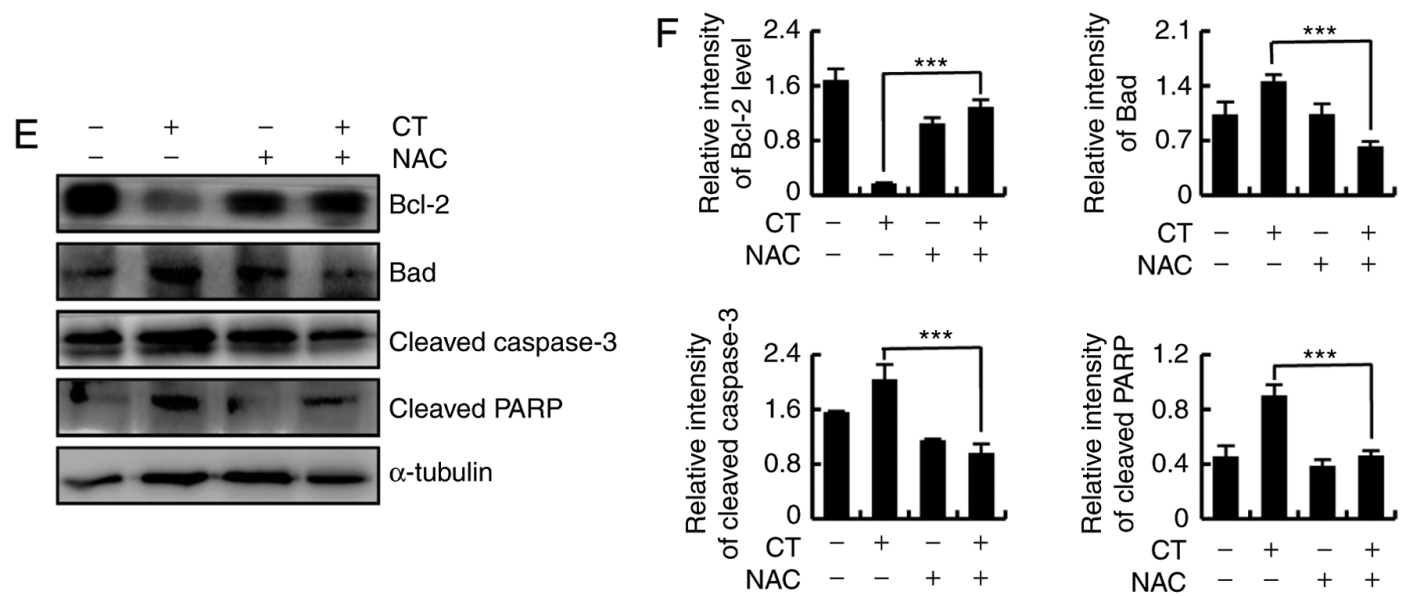

Figure 5. CT induced apoptosis via ROS-mediated signaling pathways in HFLS-RA cells. (A) Flow cytometry and (B) quantified levels of intracellular ROS in HFLS-RA cells incubated with $5 \mu \mathrm{mol} / 1 \mathrm{CT}$ for the indicated time durations $(0,3,6,12$ and $24 \mathrm{~h})$ and then stained with DCFH-DA (10 $\mu \mathrm{mol} / \mathrm{l})$ for $30 \mathrm{~min}$. (C) Flow cytometry and (D) quantification of apoptosis in HFLS-RA cells pretreated with NAC (100 $\mu \mathrm{mol} / \mathrm{l})$ for 30 min and then stimulated with $5 \mu$ mol/l CT for $24 \mathrm{~h}$. (E) Western blot analysis and (F) quantified protein expression levels of Bcl-2, Bad, cleaved caspase-3 and cleaved PARP in HFLS-RA cells treated with NAC $(100 \mu \mathrm{mol} / 1)$ and $/$ or $5 \mu \mathrm{mol} / 1 \mathrm{CT}$. ${ }^{* *} \mathrm{P}<0.01$ and ${ }^{* * * *} \mathrm{P}<0.001$, vs. untreated control group. CT, cryptotanshinone; ROS, reactive oxygen species; NAC, $\mathrm{N}$-acetyl cysteine; Bcl-2, B-cell lymphoma 2; Bad, Bcl-2-associated death promoter; PARP, poly (ADP-ribose) polymerase.

downregulated the expression of $\mathrm{Bcl}-2$, while it upregulated the protein expression levels of Bad, cleaved caspase- 3 and cleaved PARP. These results revealed that $\mathrm{CT}$ induced apoptosis through the mitochondrial pathway.

CT induces apoptosis by regulating the MAPK, Akt and STAT3 signaling pathways in HFLS-RA cells. To determine whether CT induced apoptosis by regulating the Akt, MAPK and STAT3 signaling pathways, HFLS-RA cells were treated with $5 \mu \mathrm{mol} / \mathrm{l}$ CT for different time points (0, 3, 6, 12 and $24 \mathrm{~h}$ ), and protein expression levels were detected by western blotting. As shown in Fig. 4A and B, CT exposure increased the protein expression levels of p-p38 and p-JNK, and downregulated the expression levels of p-Akt, p-ERK and p-STAT3, in a time-dependent manner. These results revealed that $\mathrm{CT}$ induced apoptosis in HFLS-RA cells via the MAPK, Akt and STAT3 signaling pathways.

CT induces apoptosis by regulating intracellular ROS levels in HFLS-RA cells. To determine whether CT induced intracellular ROS generation, the HFLS-RA cells were treated with $5 \mu \mathrm{mol} / 1 \mathrm{CT}$ for different durations $(0,3,6,12$ and $24 \mathrm{~h})$, after which the levels of ROS were detected by flow cytometry. As shown in Fig. 5A and B, CT treatment significantly increased ROS generation in a time-dependent manner.

To further investigate whether CT-induced apoptosis was mediated by intracellular ROS, the cells were divided into four groups, as follows: Control, CT, NAC and CT+NAC. As shown in Fig. 5C and D, CT-induced apoptosis was significantly suppressed after scavenging of intracellular ROS by treatment with NAC. Changes in the expression levels of apoptosis-associated proteins were detected by western blotting, which demonstrated that the expression levels of Bcl-2, Bad, cleaved caspase- 3 and cleaved PARP were recovered following intracellular ROS scavenging (Fig. 5E and F). These results indicated that the increased levels of ROS were involved in CT-induced apoptosis.

CT induces apoptosis through ROS-mediated Akt, MAPK and STAT3 signaling pathways in HFLS-RA cells. To 


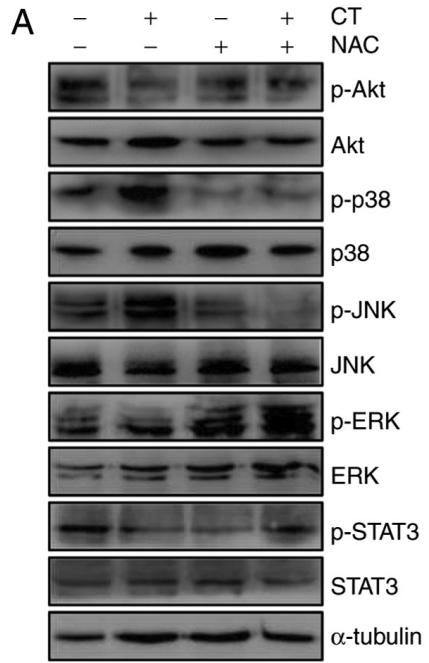

B
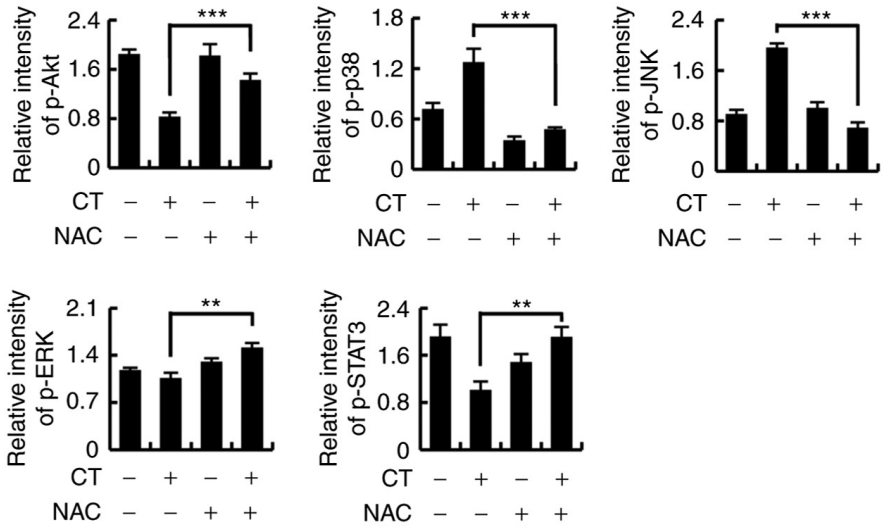

Figure 6. CT induced ROS-mediated apoptosis via the Akt, mitogen-activated protein kinase and STAT3 signaling pathways. Cells were pretreated with NAC (100 $\mu \mathrm{mol} / \mathrm{l})$ for $30 \mathrm{~min}$ and then stimulated with $5 \mu \mathrm{mol} / \mathrm{l} \mathrm{CT}$ for $24 \mathrm{~h}$. (A) Western blots and (B) quantified protein expression levels of Akt, p38, JNK, ERK and STAT3 are shown. ${ }^{* *} \mathrm{P}<0.01$ and ${ }^{* * *} \mathrm{P}<0.001$, vs. untreated control group. CT, cryptotanshinone; ROS, reactive oxygen species; NAC, N-acetyl cysteine; Akt, protein kinase B; JNK, c-Jun N-terminal kinase; ERK, extracellular signal-regulated kinase; STAT3, signal transducer and activator of transcription-3; p-, phosphorylated.

investigate whether $\mathrm{CT}$ induced apoptosis through regulating ROS-mediated Akt, MAPK and STAT3 signaling pathways, HFLS-RA cells were cultured overnight, treated with CT or NAC for $24 \mathrm{~h}$, and divided into four groups as follows: Control, CT, NAC and CT+NAC. Next, the protein expression levels were detected by western blotting. As shown in Fig. 6A and B, as compared with the CT treatment group, upregulation of p-Akt, p-ERK and p-STAT3 levels, and downregulation of p-p38 and p-JNK were detected in the CT+NAC treatment group. These data indicated that CT induced apoptosis through ROS-mediated MAPK, Akt and STAT3 signaling pathways.

\section{Discussion}

RA-FLSs are characterized by the massive imbalance between excessive cell proliferation and apoptosis, and induced cells apoptosis may be a possible treatment for patients with RA (30). Apoptosis is regulated by the mitochondrial pathway via the imbalance of pro-apoptotic (such as Bad) and anti-apoptotic proteins (such as Bcl-2) (31). Caspase-3 is the key executive mediator of apoptosis and a common downstream effector of a number of apoptotic signaling pathways (32). The present study demonstrated that the anti-proliferative and pro-apoptotic effects of CT in primary RA-FLS, HFLS-RA and MH7A cells. In addition, the results revealed that the CT-mediated apoptosis of RA-FLSs may be causally linked to the upregulation of the pro-apoptotic protein Bad and downregulation of the anti-apoptotic protein Bcl-2, as well as the activation of caspase- 3 and PARP. These findings indicated that activation of the caspase cascade contributed to the apoptosis of RA-FLSs.

The Akt signaling pathway generally restrains chondrogenesis and apoptosis mediated by Akt, and has been considered a potential target for RA treatment (33). In addition, the MAPK signaling pathway controls the proliferation, differentiation, survival and migration of various cell types, thereby serving a crucial role in cell differentiation, apoptosis, stress response, and occurrence and development of various human diseases $(34,35)$. Thus, blocking the MAPK signaling pathway as a tool for identifying a novel drug for RA treatment has been the focus of certain research studies $(36,37)$. Furthermore, the STAT3 signaling pathway is a signal transduction pathway that was identified in recent years to function in response to cytokine stimulation. It participates in numerous important biological processes, such as cell proliferation, differentiation, apoptosis and immune regulation (38). STAT3 has also been detected in the synovial lining of RA patients and animal models of RA, and STAT3 blockade promoted apoptosis in RA-FLSs (39). In the current study, the molecular mechanisms underlying CT-induced apoptosis were evaluated, and the results indicated that $\mathrm{CT}$ decreased the expression levels of Akt, ERK and STAT3, and increased the expression levels of JNK and p38. Thus, CT induced apoptosis via the Akt, MAPK and STAT3 signaling pathways in HFLS-RA cells.

ROS is a group of highly reactive molecular oxygen species produced by mitochondrial respiration, including oxygen ions, hydrogen peroxide and oxygen-free radicals (17). ROS generation is considered to be one of the primary cytotoxic mechanisms that induce cell apoptosis or necrosis (19). In normal conditions, high levels of ROS are involved in the pathogenesis of RA and may be an important mechanism for cell death (40). The results of the present study demonstrated that CT increased the levels of ROS, which led to an increase in the number of apoptotic cells, while apoptosis was suppressed by intracellular ROS scavenging. In addition, the Akt, MAPK and STAT3 signaling pathways were rescued after the scavenging of intracellular ROS. These results revealed that CT-induced apoptosis was regulated by ROS-mediated Akt, MAPK and STAT3 signaling pathways in HFLS-RA cells. In future research, the effects of CT in vivo should be evaluated.

In conclusion, the results of the present study demonstrated the mechanisms underlying the function of CT in HFLS-RA cells. Specifically, CT inhibited the proliferation and induced 
the apoptosis of HFLS-RA cells through ROS-mediated Akt, MAPK and STAT3 signaling pathways. These data provide evidence that CT may be a potential therapeutic agent for the treatment of RA.

\section{Acknowledgements}

Not applicable.

\section{Funding}

The present study was funded by the Multigrain Production And Processing Characteristic Discipline Construction Project (grant no. 2042070010), the Natural Science Foundation of Heilongjiang Province of China (grant no. LC2015036) and the Postdoctoral Scientific Research Foundation of Heilongjiang Province of China (grant no. LBH-Q13132).

\section{Availability of data and materials}

The analyzed datasets generated during the study are available from the corresponding author on reasonable request.

\section{Authors' contributions}

HNS, YHL, LQM, DJZ and CHJ conceived and designed the experiments. HNS, YHL and LQM performed the experiments. XJP, YW, JRW, HW, YiZ, JQL, WTX and YL performed the CCK-8 assay. YuZ, TZ, YHH and MHJ performed sample preparations for western blotting. GNS, YQZ and LKC performed sample preparations for flow cytometry. DJZ and CHJ wrote the paper. All authors read and approved the final manuscript.

\section{Ethics approval and consent to participate}

The present study was approved by the Ethical Committee of the Fifth Affiliated Hospital of Harbin Medical University (Daqing, China). Written informed consent was obtained from each patient involved in the current study.

\section{Patient consent for publication}

Consent for publication was obtained from the participants.

\section{Competing interests}

The authors declare that they have no competing interests.

\section{References}

1. Xu H, He Y, Yang X, Liang L, Zhan Z, Ye Y, Yang X, Lian F and Sun L: Anti-malarial agent artesunate inhibits TNF-alphainduced production of proinflammatory cytokines via inhibition of NF-kappaB and PI3 kinase/Akt signal pathway in human rheumatoid arthritis fibroblast-like synoviocytes. Rheumatology (Oxford) 46: 920-926, 2007.

2. Muthana M, Hawtree S, Wilshaw A, Linehan E, Roberts H, Khetan S, Adeleke G, Wright F, Akil M, Fearon U, et al: C5orf30 is a negative regulator of tissue damage in rheumatoid arthritis. Proc Natl Acad Sci USA 112: 11618-11623, 2015.

3. TaylorJS(ed):Thrombotic ThrombocytopenicPurpura.In:Pediatric Surgery. Springer International Publishing, Cham, Switzerland, pp303-304, 2014.
4. Firth J: Rheumatoid arthritis: Diagnosis and multidisciplinary management. Br J Nurs 20: 1179-1180, 2011.

5. Ota F, Maeshima A, Yamashita S, Ikeuchi H, Kaneko Y, Kuroiwa T, Hiromura K, Ueki K, Kojima I and Nojima Y: Activin A induces cell proliferation of fibroblast-like synoviocytes in rheumatoid arthritis. Arthritis Rheum 48: 2442-2449, 2003.

6. Kalkan A, Hallert E, Carlsson P, Roback K and Sjöwall C: Individual variations in treatment decisions by Swedish rheumatologists regarding biological drugs for rheumatoid arthritis. Scand J Rheumatol 44: 265-270, 2015.

7. Puppo F, Murdaca G, Ghio M and Indiveri F: Emerging biologic drugs for the treatment of rheumatoid arthritis. Autoimmun Rev 4: 537-541, 2005.

8. Messori A, Fadda V, Maratea D, Trippoli S, Gatto R, De Rosa M and Marinai C: Biological drugs for the treatment of rheumatoid arthritis by the subcutaneous route: Interpreting efficacy data to assess statistical equivalence. Ther Adv Musculoskelet Dis 6: 207-214, 2014

9. Tian J, Chen JW, Gao JS, Li L and Xie X: Resveratrol inhibits TNF- $\alpha$-induced IL-1 $\beta$, MMP-3 production in human rheumatoid arthritisfibroblast-like synoviocytesviamodulationofPI3kinase/Akt pathway. Rheumatol Int 33: 1829-1835, 2013.

10. Westra J and Limburg PC: p38 mitogen-activated protein kinase (MAPK) in rheumatoid arthritis. Mini Rev Med Chem 6: 867-874, 2006.

11. Hammaker DR, Boyle DL, Inoue T and Firestein GS: Regulation of the JNK pathway by TGF- $\beta$ activated kinase 1 in rheumatoid arthritis synoviocytes. Arthritis Res Ther 9: R57, 2007.

12. Valère A, Garnotel R, Villena I, Guenounou M, Pinon JM and Aubert D: Activation of the cellular mitogen-activated protein kinase pathways ERK, P38 and JNK during Toxoplasma gondii invasion. Parasite 10: 59-64, 2003.

13. Reynolds G, Cooles FA, Isaacs JD and Hilkens CM: Emerging immunotherapies for rheumatoid arthritis. Hum Vaccin Immunother 10: 822-837, 2014.

14. Migita K, Izumi Y, Torigoshi T, Satomura K, Izumi M, Nishino Y, Jiuchi Y, Nakamura M, Kozuru H, Nonaka F, et al: Inhibition of Janus kinase/signal transducer and activator of transcription (JAK/STAT) signaling pathway in rheumatoid synovial fibroblasts using small molecule compounds. Clin Exp Immunol 174: 356-363, 2013.

15. Ahmad SF, Ansari MA, Zoheir KM, Bakheet SA, Korashy HM, Nadeem A, Ashour AE and Attia SM: Regulation of TNF- $\alpha$ and NF- $\kappa$ B activation through the JAK/STAT signaling pathway downstream of histamine 4 receptor in a rat model of LPS-induced joint inflammation. Immunobiology 220: 889-898, 2015.

16. Bugatti S, Manzo A, Caporali R and Montecucco C: Inflammatory lesions in the bone marrow of rheumatoid arthritis patients: A morphological perspective. Arthritis Res Ther 14: 229, 2012.

17. Rahman N, Jeon M, Song HY and Kim YS: Cryptotanshinone, a compound of Salvia miltiorrhiza inhibits pre-adipocytes differentiation by regulation of adipogenesis-related genes expression via STAT3 signaling. Phytomedicine 23: 58-67, 2016.

18. Rahman T, Hosen I, Islam MM and Shekhar HU: Oxidative stress and human health. Adv Biosci Biotechnol 3: 997-1019, 2012.

19. Domej W, Oettl K and Renner W: Oxidative stress and free radicals in COPD-implications and relevance for treatment. Int J Chron Obstruct Pulmon Dis 9: 1207-1224, 2014.

20. Mateen S, Moin S, Khan AQ, Zafar A and Fatima N: Increased reactive oxygen species formation and oxidative stress in rheumatoid arthritis. PLoS One 11: e0152925, 2016.

21. Griendling KK, Touyz RM, Zweier JL, Dikalov S, Chilian W, Chen YR, Harrison DG, Bhatnagar A and American Heart Association Council on Basic Cardiovascular Sciences: Measurement of reactive oxygen species, reactive nitrogen species, and redox-dependent signaling in the cardiovascular system: A scientific statement from the american heart association. Circ Res 119: e39-e75, 2016.

22. Chen L, Wang HJ, Xie W, Yao Y, Zhang YS and Wang H: Cryptotanshinone inhibits lung tumorigenesis and induces apoptosis in cancer cells in vitro and in vivo. Mol Med Rep 9: 2447-2452, 2014

23. Wu CF, Klauck SM and Efferth T: Anticancer activity of cryptotanshinone on acute lymphoblastic leukemia cells. Arch Toxicol 90: 2275-2286, 2016.

24. Chen W, Pan Y, Wang S, Liu Y, Chen G, Zhou L, Ni W, Wang A and Lu Y: Cryptotanshinone activates AMPK-TSC2 axis leading to inhibition of mTORC1 signaling in cancer cells. BMC Cancer 17: 34, 2017. 
25. Chen W, Lu Y, Chen G and Huang S: Molecular evidence of cryptotanshinone for treatment and prevention of human cancer. Anticancer Agents Med Chem 13: 979-987, 2013.

26. Li XH, Yang XM and Wu XK: Effects of cryptotanshinone in lowering androgens synthesis for the prenatally androgenized male rats. Zhongguo Zhong Xi Yi Jie He Za Zhi 28: 1001-1004, 2008 (In Chinese).

27. Cha JD, Lee JH, Choi KM, Choi SM and Park JH: Synergistic effect between cryptotanshinone and antibiotics against clinic methicillin and vancomycin-resistant staphylococcus aureus. Evid Based Complement Alternat Med 2014: 450572, 2014.

28. Li W, Saud SM, Young MR, Colburn NH and Hua B: Cryptotanshinone, a Stat3 inhibitor, suppresses colorectal cancer proliferation and growth in vitro. Mol Cell Biochem 406: 63-73, 2015.

29. Lin L, Wang D, Li L, Ding X and Ma H: Dehydroepiandrosterone inhibits cell proliferation and improves viability by regulating $S$ phase and mitochondrial permeability in primary rat leydig cells. Mol Med Rep 14: 705-714, 2016.

30. Ye T, Zhu S, Zhu Y, Feng Q, He B, Xiong Y, Zhao L, Zhang Y, $\mathrm{Yu} \mathrm{L}$ and Yang L: Cryptotanshinone induces melanoma cancer cells apoptosis via, ROS-mitochondrial apoptotic pathway and impairs cell migration and invasion. Biomed Pharmacother 82: 319-326, 2016.

31. Du J, Chen C, Sun Y, Zheng L and Wang W: Ponicidin suppresses HT29 cell growth via the induction of G1 cell cycle arrest and apoptosis. Mol Med Rep 12: 5816-5820, 2015.

32. Zhou L, Luan H, Dong X and Li Y: Activation of the PI3K/Akt and MAPK signaling pathways antagonizes adriamycin-induced HL-60 leukemia cell apoptosis. Mol Med Rep 3: 641-644, 2010.

33. Li S, Chen JW, Xie X, Tian J, Deng C, Wang J, Gan HN and Li F: Autophagy inhibitor regulates apoptosis and proliferation of synovial fibroblasts through the inhibition of PI3K/AKT pathway in collagen-induced arthritis rat model. Am J Transl Res 9: 2065-2076, 2017.
34. Zhai H, Hu S, Liu T, Wang F, Wang X, Wu G, Zhang Y, Sui M, Liu $\mathrm{H}$ and Jiang L: Nitidine chloride inhibits proliferation and induces apoptosis in colorectal cancer cells by suppressing the ERK signaling pathway. Mol Med Rep 13: 2536-2542, 2016.

35. Hao W, Zhang X, Zhao W, Zhu H, Liu ZY, Lu J and Chen X: Cryptotanshinone induces pro-death autophagy through JNK signaling mediated by reactive oxygen species generation in lung cancer cells. Anticancer Agents Med Chem 16: 593-600, 2016.

36. Park KR, Yun HM, Quang TH, Oh H, Lee DS, Auh QS and Kim EC: 4-Methoxydalbergione suppresses growth and induces apoptosis in human osteosarcoma cells in vitro and in vivo xenograft model through down-regulation of the JAK2/STAT3 pathway. Oncotarget 7: 6960-6971, 2016.

37. Cheng G, Gao F, Sun X, Bi H and Zhu Y: Paris saponin VII suppresses osteosarcoma cell migration and invasion by inhibiting MMP-2/9 production via the p38 MAPK signaling pathway. Med Rep 14: 3199-3205, 2016.

38. Miao D and Zhang L: Leptin modulates the expression of catabolic genes in rat nucleus pulposus cells through the mitogen-activated protein kinase and Janus kinase 2/signal transducer and activator of transcription 3 pathways. Mol Med Rep 12: 1761-1768, 2015.

39. Yang Y, Dong Q and Li R: Matrine induces the apoptosis of fibroblast-like synoviocytes derived from rats with collageninduced arthritis by suppressing the activation of the JAK/STAT signaling pathway. Int J Mol Med 39: 307-316, 2017.

40. Zheng R, You Z, Jia J, Lin S, Han S, Liu A, Long H and Wang S: Curcumin enhances the antitumor effect of ABT-737 via activation of the ROS-ASK1-JNK pathway in hepatocellular carcinoma cells. Mol Med Rep 13: 1570-1576, 2016. 